\title{
PUDRICION NEGRA EN RAICILLAS DE PALTO (Persea americana Mill.) POR Cylinatrocarpon destructans: PATOGENICIDAD Y ASPECTOS EPIDEMIOLOGICOS
}

\section{Black root rot in avocado plants (Persea americana Mill.) by Cylindrocarpon destructans: pathogenicity and epidemiological aspects}

\author{
Ximena Besoain C. ${ }^{1} \&$ Eduardo Piontelli L. ${ }^{2}$ \\ ' Facultad de Agronomía. Universidad Católica de Valparaiso. Casilla 4 - D. Quillota. \\ ${ }^{2}$ Cátedra de Micología. Facultad de Medicina. Universidad de Valparaíso. Casilla 92 - V. Valparaíso.
}

Palabras clave: Pudrición negra. Persea americana. Cylindrocarpon destructans, patogenicidad. epidemiología Key words: Black root rot. Persea americana, Cylindrocarpon destructans, pathogenicity, epidemiology

\section{RESUMEN}

En un vivero comercial de Quillota (I Región. Chile), durante los años $199+$ y 1995 se produjo en plantas de palto (Persea americana Mill.), un suibito decaimiento de éstas, caracterizado por sintomas iniciales de marchitez, pérdicla de turgor de las hojas, asociada a clorosis v necrosis marginaly al mismo tiempo a nivel de las raices, se observin una pudrición severa de color café oscuro a negro. Sólo por concepto de estos problemas, se destruyeron 22.000 plantas en dos años. A partir de la zona de avance de las lesiones radiculares, se aistó en forma consistente colonias de Cylindrocarpon destructans, no detectándose representantes del género Phytophthora. Con el propósito de determinar la posible fuente de inóculo, se efectuaron aislamientos desde el sustrato empleado, sus diferentes componente y de semillas. recuperándose este patógeno sólo desic la arena de rio y suelo de tranque.

De acuerdo con los resultados obtenidos a partir de los diferentes aislamientos, las pruehas de patogenicidad y los sintomas observados, se considera a C: destructans como el agente causal de una mueva enfermestad que afecta a plantas de palto en vivere.

\section{INTRODUCCION}

Durante los años $1994-1995$ se observó en plantas de palto ( Persea americana Mill. ) producidas a nivel de virero comercial. síntomas iniciales de marchitez observándose un rápido colapso de las hojas apicales. con

\section{SUMMARY}

During $199+$ and 1995 a severe decay was observed affecting potted plants of arocado (Persea americana Mill.) grown at a commercial mursery, located in Ouillota, I Region of Chile. The decav consisted of a suddenly dieback of the plants, showing signs of loss of turgor in the leaves associated to a marginal chlorosis and necrosis whereas a severe brownish to blackish rot could he observed at the level of the roots. This disease caused in tro years the death of more than 22.000 potted plants. From the basis of the lesions present in the roots, fungal colonies of Cylindrocarpon destructans were consistenty isolated. while no representatives of the genera Phytophtora were detected. With the purpose of determining the possible source of inoculations. isolates fiom the substrate, it different components and its seeds were made, concluding that this pathogen could only be detected in the sand from river and the soil of water reservoir: Considering the results of the different isolates, pathogenicity test and singns observed, it is demostrated that $C$. destructans is the causing agent of a nen disease affecting anocado plants grown under nursery conditions.

un clecaimiento regresivo de las plantas en la parte aérea, debiclo a pérdida de turgor de las hojas (síntoma de un «quitasol cerrado») (Fig.1A). Al observar el sistema radicular se visualizaron síntomas de pudrición de color oscuro a nivel de raices y raicillas (Fig. IB). Presentados 
los primeros síntomas en la parte aérea. cl desarrollo de la enfermedad fue relativamente rápido. produciéndose la muerte de 20 a $25 \%$ de las plantas al cabo de dos a tres meses ( 22.000 plantas en dos años).

Esta patología fue inicialmente confundida con tristeza del palto. cuyo agente causal es Plytophthora cinnamomi Rands. Sin embargo. se descartó la relación con la enfermedad antes descrita, debido a que los sintomas aéreos difieren. ya que esta produce: clorosis. disminución del tamaño foliar y un decaimiento más lento en las plantas afectadas a nivel de vivero. Además, a partir de las raices provenientes de árboles afectados, no se aisló ninguna especie de Phytophthora, incluso estrategias de control químico normalmente empleadas para esta patología. no cjercieron ningún efecto.

De acuerdo con los análisis fitopatológicos efectuados a plantas afectadas, realizados en el Laboratorio de Fitopatología de la Facultad de Agronomía de la Universidad Católica de Valparaíso. se aisló en forma consistente a partir de raicillas enfermas. estructuras reproductivas correspondiente al género Cylindrocarpon (C.destructans). el que de acuerdo con antecedentes bibliográficos, es capaz de causar pudrición de raíces («root rot») en diferentes hospedadores de los géneros: Abies, Aster. Beta, Fragaria, Ginseng, Juglans. Lilium, Malus, Narcisus. Pinus. Prumus; Rhododendrum, Solanum, litis, entre otras (Coyier \& Roave. 1988; Daughtrey et al., 1995; Domsch et al., 1980: Grasso, 1984: Matua \& Miyazawa. 1984: Montecchio \& Causin. 1995: Ogawa. et. al., 1995). En la bibliografia chilena. sólo figura la presencia de $C$. destructans afectando raíces y cuello de plantas de vivero de Pinus radiata (Andrade, 1996).

No obstante lo antes mencionado, el género Cylindorcarpon se encuentra asociado a raíces en otras especies vegetales, siendo un habitante común de la rizósfera, es decir, sin causar en estos hospedadores enfermedad (Campbell, 1985: Dahm \& Strzelczyk, 1987). Por lo tanto, para determinar su asociación a decaimiento en plantas de palto, se efectuaron pruebas de patogenicidad .

Otro aspecto importante que consideramos. fue la posible fuente de inóculo de este microorganismo. analizando especialmente los diferentes componentes del sustrato empleado y las semillas.

Por las razones anteriormente cxpuestas, nuestros objetivos fueron:

-Aislar el agente causal a partir de plantas enfermas, semillas de palto y de muestras del sustrato y sus componentes empleados para la producción de plantas en macetas.

-Efectuar pruebas de patogenicidad en plantas de palto aparentemente sanas, inoculadas con tres diferentes aislamientos de $C y$ lindrocarpon sp. y determinar la especie involucrada.

\section{METODOLOGIIA}

Este trabajo fue realizado obteniendo material vegetal enfermo y sustrato desde el vivero de la Estación Experimental La Palma. perteneciente a la Facultad de Agronomía de la Universidad Católica de Valparaíso.

\section{1.- Medios de cultivo}

Para los diferentes aislamientos efectuados, se emplearon los siguientes medios de cultivos: Agar Papa Dextrosa acidificado a pH 5.5 (APDA), Agar Extracto Malta (AEM). Medio Selectivo para Fusarium (MSF) ( Nash \& Snyder (1962) citado por TSAO (1970)). Agar Czapeck's (CZA) y Agar Spezicller Nährstoffarmer, más extracto de levadura (SNAY), todos medios empleados para el aislamientos de especies del género $C y$ lindrocarpon (Brayford. 1992 ).

\section{2.- Aislamiento desde raíces}

Para aislar al agente causal a partir de raíces enfermas, se seleccionaron plantas de palto en bolsa con diferentes grados de intensidad de ataque, algunos con sintomas de clorosis, $y$ otros con clara marchitez del brote apical en la parte aérea. A partir de la zona de avance de las lesiones observadas en las raices, se extrajeron trozos de I a $1.5 \mathrm{~cm}$ de largo. los que fueron superficialmente desinfectados por 15 segundos en una solución de hipoclorito de sodio al $1 \%$. lavadas profusamente en agua destilada estéril (ADE), secadas y luego sembradas en placas con APDA y MSF. Una vez obtenido el crecimiento micelial, se procedió a aislar colonias esporuladas del hongo a placas con APDA con el propósito de obtener colonias individuales. las que fueron subcultivadas a partir de los ápices de las hifas. Los cultivos puros, mantenidos a $5^{\circ} \mathrm{C}$, fueron conservados en tubos de ensayo con ADE y SNAY.

\section{3.- Aislamiento desde las semillas}

Para el aislamiento de especies de $C y$ lindrocarpon a partir de semillas, se procedió a la obtención de éstas desde el material empleado como portainjertos a nivel de vivero, las que correspondian a la variedad Mexícola. Para el aislamiento se empleó placas con APDA, AEM y MSF, sembrándose trozos tanto de la testa como del endosperma, con y sin esterilización previa con hipoclorito de sodio al $1 \%$ por un minuto.

\section{4.- Aislamientos desdle el sustrato y sus componentes}

Se efectuaron aislamientos a los diferentes componentes del sustrato, arena de río. acícula de pino, suelo de tranque nuevo ( extraido recientemente ), suelo de tran- 
que antiguo ( suelo utilizado el año anterior ), arena rubia más aserrín, y dos mezclas, una elaborada recientemente (arena de río. tierra de hojas, suelo de tranque y acícula de pino) $(1: 1: 1: 1 \mathrm{v} / \mathrm{v} / \mathrm{v} / \mathrm{v})$ (sin desinfección) y la otra realizada cl año anterior ( esterilizada con vapor). En todos se cmpleó un método de dilución ( $1 \times 10^{-3}$ y $1 \times 10^{-1} \mathrm{ml} / \mathrm{g}$ de suelo) en tres medios selectivos APDA modificado con $100 \mathrm{ppm}$ de streptomicina, MSF y AEM.

\section{5.- Prueba de Patogenicidad}

Para la realización de la prueba de patogenicidad se procedió a obtener plantas sanas de palto, eligiéndose portainjertos de tres meses de desarrollo y que no presentaron signos visibles de pudrición a nivel de las raices o raicillas. Las plantas elegidas correspondieron a la variedad Mexícola, cuyas semillas habían sido preliminarmente analizadas como libres de patógenos, especialmente del género $C y$ lindrocarpon. Se utilizó el mismo sustrato anteriormente empleado en la formación de portainjertos, con el propósito de asegurar el desarrollo de la enfermedad, al no alterar las condiciones de humedad ni de pH.

Las plantas se mantuvieron bajo condiciones de sombreadero, siendo inoculadas con dos aislamientos obtenidos desde arena (7 A-1 y 7 A-3) y uno desde suelo de tranque ( $7 \mathrm{~A}-4)$, más un cuarto aislamiento obtenido a partir de raíces de palto ( 8-1). La metodología de inoculación consistió en la multiplicación del inóculo en placas con APDA, las que fueron incubadas a $22^{\circ} \mathrm{C}$ por 7 días, procediéndose a extraer el micelio y conidios e incorporarlo en ADE, luego se homogenizó en una licuadora hasta obtener una concentración de $1 \times 10^{6}$ propágulos $/ \mathrm{ml}$. incorporándose $100 \mathrm{ml}$ de esta solución al sustrato de cada planta a inocular. Se inocularon 6 plantas por cepa dejándose 6 plantas sin inocular como testigo. Luego de ser inoculadas, las plantas fueron mantenidas bajo condiciones de sombreadero hasta el desarrollo de síntomas. La evaluación se efectuó en tres plantas a los dos meses post inoculación, efectuándose reaislamientos a partir de raíces dañadas. La segunda evaluación se efectuó a los ocho meses post - inoculación en las tres plantas restantes de cada tratamiento.

\section{6.- Identificación de la especie de $C$ ylindrocarpon.}

Una vez obtenidas las cepas puras de Cylindrocarpon, se procedió a su estudio en APDA, CZA y SNAY. Se consideraron las siguientes características:

a) Macroscópicas en APDA y CZA (textura de las colonias, color de anverso- reverso, pigmentos, diámetro de crecimiento a los 10 días a $25^{\circ} \mathrm{C}$ ).

b) Microscópicas en SNAY (forma - tamaño de las células conidiógenas, macro, microconidios y clami- dosporas). Estas últimas características morfométricas, se basaron en un mínimo de 20 mediciones al azar por cada estructura analizada, basándonos en los trabajos de Booth, 1966; Samuels \& Brayford, 1990; Brayford, 1992.

\section{RESULTADOS Y DISCUSION}

La sintomatología de este nuevo problema se expresa inicialmente con una menor tasa de crecimiento de las plantas, las que se vuelven cloróticas, con marchitamiento de las hojas superiores y muerte regresiva en la parte aérea de las plantas (Fig.1.A). Estos problemas son indirectamente producidos como consecuencia de una pudrición severa de raíces, siendo afectadas inicialmente las raicillas, tanto la corteza como el cilindro vascular, abarcando posteriormente a todo el sistema radical (Fig. 1B).

\section{1.- Aislamientos desde raíces}

A partir de la zona de avance de las lesiones en las raíces, se aisló en forma consistente colonias de Cylindrocarpon. Este aspecto, unido a la total ausencia del género Phytophthora en las raíces dañadas (aunque se utilizó en forma paralela un medio selectivo para su aislamiento ( P10 PV) (Tsao, 1970), permitieron descartar que la patología observada correspondía a un ataque de tristeza del palto. Además, la especie Phytophthora cinnamomi Rands, se caracteriza por causar inicialmente pudrición sólo a nivel de la corteza de las raíces. Tampoco fue observado un pardeamiento o estrías necróticas a nivel del tẹiido xilemático. En Sudáfrica, se reportó la presencia de especies de este género afectando plantas de palto cv. Hass, injertadas sobre los portainjertos Duke 7 y G755, donde las plantas presentaron una pudrición severa de sus raíces, sin embargo, se observó que plantas con adecuado nivel de N y P se mantenían sanas (Barnard, 1988).

La total descomposición de los tejidos de la raíz, tanto de la corteza como estela, concuerda con estudios realizados en frejol, en donde se demostró que la estela y la corteza de la raíces son frecuentemente colonizadas por Cylindrocarpon sp. (Campbell, 1985). Este mismo autor describe otro estudio realizado con raíces apicales de $V i$ cia faba, donde $C$. destructans demostró ser un colonizador primario.

\section{2.- Aislamiento desde semillas}

$\mathrm{Al}$ analizar los tejidos tanto externos como internos, no se detectó la presencia de colonias correspondientes al género $C y$ lindrocarpon. Es importante aclarar que algunas semillas fueron obtenidas de frutos recojidos del suelo; y por lo tanto, las semillas podrían haber sido colonizadas por este patógeno cosmopolita de habitat geofílico (Domsch et al., 1980). 


\section{3.- Aislamientos dlesde sustratos y sus componentes}

A partir de los 21 aislamientos efectuados a los diferentes componentes del sustraio. sólo se aislaron colonias de $C$. destractain $\mathrm{cu}$ arena de río y suclo de tranquue nucio. Esto. resulta lógico al analizar los factores que predisponen cl desarrolio de las especies correspondientes a este género. las gue prefieren condiciones de cxceso de agua (Daughtrey et al., 1995). situación común en arena de río y en un suclo proveniente de tranque. especialmente en este último. por sus condiciones de salturación de agua y anacrobiosis. Es interesante destacar la ausencia de este género en el sustrato compuesto por acículas de pino, incluso en los aislamientos efectuados con siembra directi. aunque C. destructans. comummente es capaz de colonizar la rizosfera de especies de pino (Andrade. 1996. Unestam. 1995).

Cabe destacar. que no fue posible aislar colonias de Cylindrocurpon en mezcla de suclo nueva o antigua. siendo que plantas desarrolladas en este sustrato presentaron la enfermedad. Esto podría explicarse en base a que la metodologia compleada sólo es capar de detectar poblaciones cercanas o superiores a $1(0)(0)$ u.f.c./ ml.

\section{4.- Prueba de Patogenicidad en plantas}

Esta prucba fue realizada durante los meses de octubre de 1995 y enero de 1996. Las plantas se establecieron bajo condiciones de sombreadero con el propósito de que la temperatura del sustrato fuera moderada y existicsen condiciones para el desarrollo de la enfermedad. Además. con este mismo objetivo. las plantas fueron regadas constantemente cada dos a tres clias. asegurándose de este modo condiciones de saturación de agua en el suelo.

Al cabo de 30 días de inoculadas las plantas. algunas de ellas comenzaron a mostrar síntomas de clorosis y menor crecimiento. en comparación con el tratamiento testigo. Al cabo de 62 días post - inoculación se procedió a la evaluación del daño a nivel del sisiema radical, existiendo un notorio daño en plantas inoculadias con algunas cepas. como la cepa $7 \mathrm{Al}$ ( Fig. IE). cn comparación con cl tratamiento testigo. La cepa 7A-4 obtenida desde suelo de tranque fue la que presentó en forma más homogénea el mayor daño a nivel de ráces (Cuadro 1 ).

Para posteriores evaluaciones se dejaron tres. plantas correspondientes a cada uno de los cuatro tratamientos efectuados. observándose a los ocho meses de inoculadas síntomas de pérdida de turgor de las hojas (Fig. IF). existiendo en las plantas inoculadas un daño severo en todo el sistema radicular. apreciandose éste de color café oscuro a negro. Barmard (1989). asocia problemas de pudrición severa de raices en plantas de palto por Cylin- drocarpon spp... al estudiar el efecto de plantas cv. Hass injertadas sobre Duke7 y G755 cn macelas con solución Hougland. eliminando en éstas la presencia de macro o micronui rientes.

CUADRO 1. Patogenicidad de $\&$ cepas de $C$. destructans, inoculadas en aráces de plamtas de palito *

\begin{tabular}{|lcc|}
\hline Tratamiento & $\begin{array}{c}\text { Sindaño } \\
\text { o leve }\end{array}$ & $\begin{array}{c}\text { Moderado } \\
\text { a severo }\end{array}$ \\
\hline Testigo & $3 / 3$ & - \\
$7 \mathrm{~A} 1$ & $1 / 3$ & $2 / 3$ \\
$7 \mathrm{~A} 3$ & $1 / 3$ & $2 / 3$ \\
$7 \mathrm{~A} 4$ & - & $3 / 3$ \\
$8 \mathrm{~A} 1$ & $1 / 3$ & $2 / 3$ \\
\hline
\end{tabular}

(*) Se evaluó un total de tres plantas por tratamiento at los dos meses post-imoculacion

Es importante señalar que el efecto patogénico de esta especie. se debe a una colonización directa de los tẹidos radicales produciendo pudrición de raicillas y raíces. Tambićn se ha scĩalado un efecto indirccto, como demostraron en 1967. Cartwright y IVhite (en Ashtony (Villis 1982). quienes aislaron desde cultivos puros de $C$. destructans un compuesto fitotóxico. denominado Nectrolide. capaz de detener el crecimiento y ennegrecer las raices de Eucalyptus. Por otro lado, Dahm \& Strzclczyk (1987). destacan a C. destructums, como un hongo pionero en la colonización de raices. y aunque no siempre es un patógeno fucrte. ha sido demostrada su patogenicidad en numerosas especies forestales. frutales y ornamentales (Andrade. 1996: Coyier \& Roanc. 1988; Dahm \& Strzelczyk, 1987: Grasso. 1984: Matua \& Miyazalwa. 1984: Unestam et al., 1989).

Con esta prueba queda demostrada la patogenicidad de cepas de $C$. destructans, al causar daño en plantas de palio cultivadas en macetas.

\section{5.- Identilicación de la especie de Cy/indrocarpon}

Los + grupos de Cylindrocarpon descritos por Booth (1966). a pesar de no ser naturales, permitieron en forma prácticia una buena ubicación de nuestras cepas dentro del grupo 3. Se pensó inicialmente.en Cylindrocarpon didymum más que en C. destructans (Besoain \& Piontelli. 1997). por sus características macroscópicas (crecimiento y color de las colonias). sus macroconidios. preclominantemente con I septo en los cultivos vicjos y la ausencia inicial de clamidosporas. Sin embargo, basándonos posteriormente en las observaciones efectuadas por Samuels \& Brayford $(199())$. referentes a las variaciones observadas en Nectria radicicola y sus anamorfos (C.destructans). comparamos nuest ros aislamientos con las 5 va- 


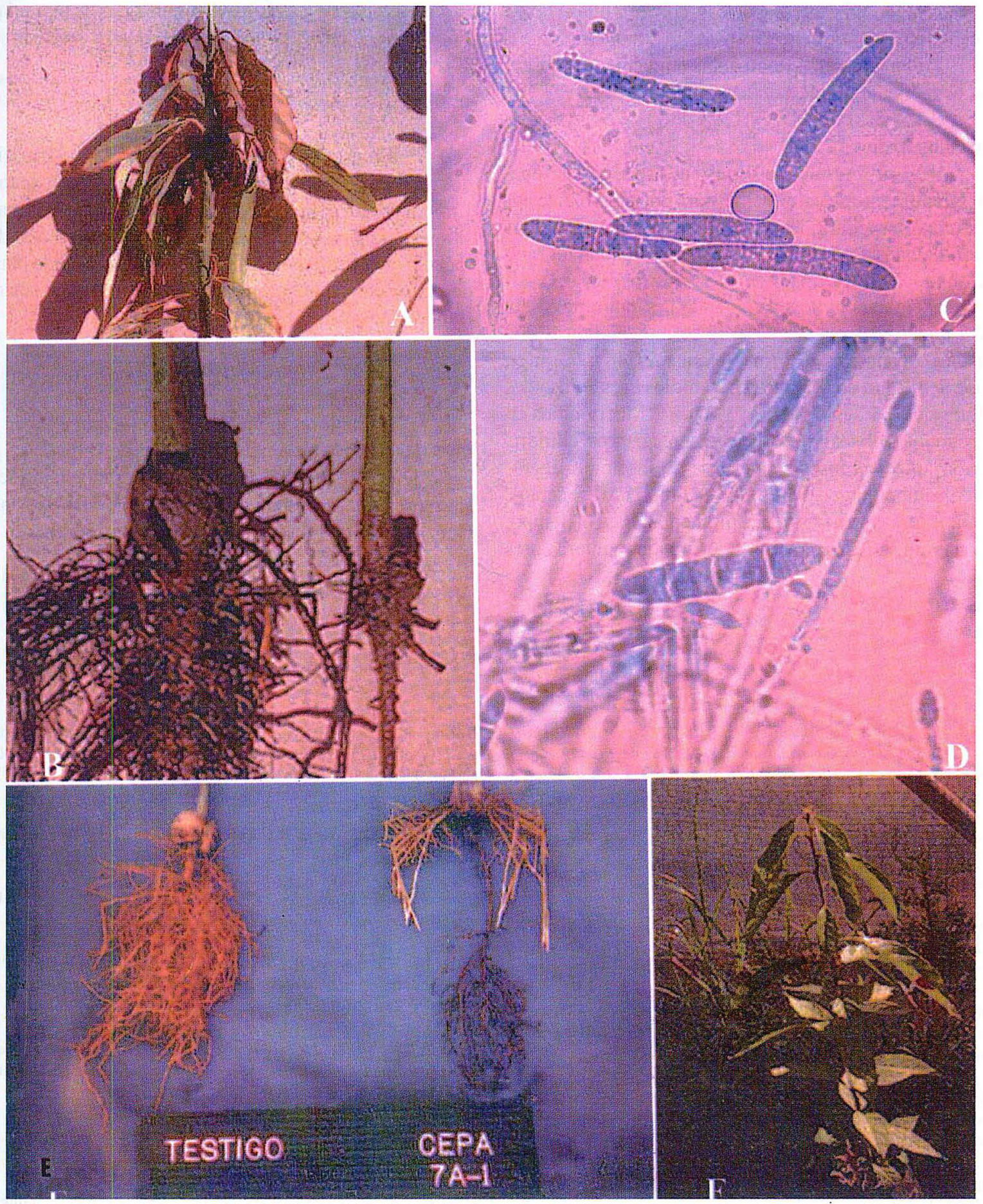

Figura 1. A. Planta de palto en maceta afectada por C.destructans. B. Pudrición negra severa de raíces observada en plantas de vivero. C. Macroconidias de $C$. destructans. D. Conidióforos, microconidios y macroconidias de $C$. destruct tans. E. Raíces de planta de palto inoculadas (2 meses)con $C$. destructuns (cepa 7 A-1), en comparación a planta testigo (izq.). F. Planta de palto expresando síntomlas en parte aérea (8 meses post-inoculación). 
riantes morfológicas descritas. La morfología de los macroconidios en cultivos frescos ( predominantemente con 3 septos (Fig C y D), permitieron junto a todas las otras características macro y microscópicas, concluir que el taxon correspondía a $C$. destructans (Zinss.) Scholten.

Todas las cepas analizadas e inoculadas presentaron la siguientes características morfológicas:

Colonias en APDA y CZA de 34 - 62 mm de diámetro a los 10 días a $25^{\circ} \mathrm{C}$.

En APDA, micelio flocoso de aspecto aterciopelado, inicialmente de color beige, para posteriormente adquirir una coloración café rojiza. El reverso de las colonias con tonalidades desde el beige al café oscuro (con las mismas tonalidades en CZA).

Células conidiógenas abundantes, cilíndricas, derechas, que se adelgazan hacia el ápice, monofialídicas, en verticilios o dispuestas en forma irregular, $21-48 \times 2-3 \mu \mathrm{m}$ en la base, 1,5- $2 \mu \mathrm{m}$ en el ápice. Micronidios lisos, elipsoides a cilíndricos 0-1 septo, $4-9 \times 3-4 \mu \mathrm{m}$. Macronidios en esporodoquios, lisos, rectos a levemente curvos, cilíndri$\cos$, con ápices obtusos y una base de inserción ligeramente protuberante, 28 y $36 \times 5-6 \mu \mathrm{m}$, con 1-3 septos. Clamidosporas globosas, lisas, pigmentadas en el tiempo, solitarias, en cadenas o racimos, terminales o intercalares
$10-12 \mu \mathrm{m}$.

Las características morfológicas observadas en nuestras cepas, concuerdan en gran parte con las observadas en cepas de $C$. destructans aisladas en plántulas de Pinus radiata por Andrade (1996), en la IX Región.

\section{CONCLUSIONES}

En este estudio quedó demostrada la capacidad de cepas de $C$. destructans, de causar pudrición de raíces y raicillas y un daño generalizado en las plantas de palto.

Los componentes que estarían incorporando el inóculo al sustrato empleado en la producción de plantas de palto, corresponden a la arena de río y al suelo de tranque (nuevo).

\section{AGRADECIMIIENTTOS}

Los autores agradecen el financiamiento de esta investigación a la Universidad Católica de Valparaíso , proyecto DGI $242.768 / 95$; la asistencia técnica a Francisca Herrera, Ximena Casanueva y Gladys Andrade, y a Mónica Gonzalez por la transcripción de este manuscrito.

\section{REFERENCIAS}

Andrade, O. (1996).Identificación de Cylindrocarpon destructans (Zinss.) Scholten asociado a plantas de 1-2 años de Pinus radiata D. Don afectadas por pudrición de raices y tallos. Fitopatologia (resumen) 31:18

Ashton, D.H. \& Willis, E. J. (1982). In: The Plant Community as a working Mechanism. E. Y. Newmann (Ed.). Spec. Publ. Serv. Br. Ecol. Soc. pp. $111-128$

Barnard, R.O. (1988): Nutrient eliminatin treatments with potted avocado plants. Yearbook, South African Avocado Grower's Association. 11:25-26

Besoain,X. \& Piontelli, E. (1997). Pudrición negra de raicillas en palto (Persea americana Mill.)causada por Cylindrocarpum didymum (affinis). VII.Congreso Chileno de Fitopatologia.

Brayford, D. (1992). Cylindrocarpon. In: Methods for research on soilborne phytopathogenic fungi. 103-106. APS Press, St. Paul, Minnesota. U.S.A.

Campbell, R. (1985). Plant Microbiology. Ed. Arnold, Baltimore, Maryland, U.S.A.

Coyiero D. L. \& Roane, M. K. (1988). Compendium of Rhododendrum and azaleas diseases. 2 nd. Ed., APS Press, St. Paul, Minnesota.
Dahm, H. \& Strzelczyk, E. (1987). Cellulolytic and pectolitic activity of Cilindrocarpon destructans (Zins.) Scholt. Isolates pathogenic and non-pathogenic to fir (Abies alba Mill. ) and Pine (Pinus sylvestris L.). J. Phytopathology 118:75-83

Daughtrey, M.; Wick, R.; Petrson, J. (1995). Compendium of flowering potted plant disease. APS Press, St. Paul Minnesota.

Domsch, K.H.; Gams, W. \& Anderson, T. (1980). Compendium of soil fungi. Academic Press, London.

Ellis, M.; Conserve, R.; Williams, R.; Williamson, B. (1991). Compendium of strawberry and blackberry diseases and insects. APS Press, St. Paul Minnesota.

Grasso, S. 1984. Infezioni de Fusarium oxysporum e di Cylindrocarpon destructans a una moria de giovani piante de vite in Sicilia. Informatore Fitopatologico 34:59-62

Mass, J. (1992). Compendium of Strawberry diseases. 3erd. Ed APS Press, St. Paul, Minnesota.

Matuo, T. \& Miyazawa, Y. (1984). Scientific name of Cylindrocarpon sp. causing root rot of Ginseng. Ann. Phytopath. Soc. Japan 50:649-652

Montecchio, L. \& Causin, R. (1995). First Report of 
Cylindrocarpon destructans on English Walmut in Italy. Plant Disease 79:967

Ogawa, J.; Zehr, E.; Bird, G.: Ritchie, D.; Uriu, K. ; Uyemoto, J. (1995). Compendium of stone fruit diseases. APS Press. St. Paui, Minnesota.

Rossman, A. Y: Palm, M.E. \& Spiclman, L.J. (1990). A literature for the identification of plant pathogenic fungi. 2 nd. De. APS
Press, St. Paul, Minnesota.

Unestam, T.; Beyer-Ericson, L \& Strand, M. (1989). Involvement of Cylindrocarpon destructans in root death of Pinus sylvestris seedling pathogenic behavior and predisposing factors. Scann. J. For. Res, 4:521-536

Tsao, P.H. (1970). Selective media for isolation of pathogenic fungi. Annu. Rev. Phytopathology 8:157-186 